Journal of Information Systems Engineering

\& Management, 2(3), 14

\title{
Brachial Plexus Blocker Prototype
}

\author{
Stéphanie Coelho Monteiro ${ }^{1 *}$ \\ ${ }^{1}$ Escola Superior de Tecnologia e Gestão Instituto Politécnico de Bragança, PORTUGAL
}

*Corresponding Author: ste.cmonteiro@hotmail.com

Citation: Monteiro, S.C. (2017). Brachial Plexus Blocker Prototype. Journal of Information Systems Engineering \& Management, 2(3), 14. doi: 10.20897/jisem.201714

Published: August 2, 2017

\begin{abstract}
Although the area of surgical simulation has been the subject of study in recent years, it is still necessary to develop artificial experimental models with a perspective to dismiss the use of biological models. Since this makes the simulators more real, transferring the environment of the health professional to a physical or virtual reality, an anesthetic prototype has been developed, where the motor response is replicated when the brachial plexus is subjected to a proximal nervous stimulus. Using action-research techniques, with this simulator it was possible to validate that the human nerve response can be replicated, which will aid the training of health professionals, reducing possible risks in a surgical environment.
\end{abstract}

Keywords: anesthesia, simulation, brachial plexus blockade, Arduino, 3D print

\section{CONTEXT AND THEORETICAL FOUNDATION}

Regional anesthesia has always required deep anatomical knowledge, especially of surface anatomy, so it is possible to infer the location of deep structures based on palpable points of reference, such as bony prominences and muscular borders. Anesthetic techniques for peripheral nerve blocking have evolved from the most conventional ones, such as the search for paresthesia and the loss of resistance, to the more modern ones, such as nerve stimulation and ultrasonography. Today, the search for paresthesia is questioned by most anesthesiologists, since the mechanical stimulation of the needle in the nerve can be harmful and uncomfortable for the patient.

In electrical nerve stimulation, a current of $0.5 \mathrm{~mA}$, provoking an appropriate motor response, is considered acceptable, suggesting that the needle is sufficiently close to the nerve. Studies show that the motor response to the electrical stimulus in the nerve has low sensibility to the neural localization, and it can be inferred that the neurostimulator needle can come into contact with the nerve to be blocked without resulting in motor stimulus, decreasing the safety of the anesthetic procedure. In this case, the ultrasound image allows the exact visualization of the needle as it approaches the nerve, avoiding such undesirable contact.

Today, the new trend is to perform the blockade of peripheral nerve guided by ultrasound, visualizing in real time the insertion of the needle, its relation with the adjacent structures the tissue plan of deposition of the local anesthetic and its subsequent dispersion.

The maintenance of a distance, in which only the local anesthetic will come into direct contact with the nerve, transforms the trauma through the needle and the intraneural injection in avoidable lesions reveals a less aggressive conduct, prevents uncomfortable sensations to the patient and adds more safety to the anesthetic procedure (Backus et al., 2016).

Anesthesiology deals with situations that require immediate responses because of critical situations and it is therefore important to develop improvement techniques. Ultrasound has been increasingly used in peripheral blockade. Ultrasound-guided techniques are based on direct visualization of the nerves, the locking needle and the adjacent anatomical structures. This visualization of the structures by means of ultrasound with high frequency 
transducers provides the anesthesiologist with the security of the correct positioning of the needle and the monitoring of the distribution of the local analgesic in real time, thus improving the quality of the block and increasing the advantage of avoiding traumatic neurological complications. The possibility of complications when performing anesthetic blockades justifies the use of ultrasonic assistance, which must be developed before blocking patients. Hence the importance of simulators and drills in experimental models. The experimental models currently used for ultrasound-guided blocking are performed on pork shoulders, chicken breasts, bovine muscles or gelatin pieces (Carvalho, 2016).

The importance of training in simulation has been considered over the years with the creation of simulators adapted to reality and meeting the needs of healthcare professionals. Proper training allows for quick responses, skill development, and skill enhancement with the material concerned, thereby reducing the risk to the patient.

There are several types of anesthesia: i) general anesthesia, ii) regional anesthesia, iii) sedation/ monitored anesthetic care, and iv) local anesthesia. In peripheral nerve blocks, the local anesthetic is administered around the nerves responsible for the sensitivity and movement of the target limb of surgery. For example, for hand surgery, it is possible to anesthetize only the arm, by administering local anesthetic at the level of the armpit.

Brachial plexus block is an anesthetic technique used for upper limb surgeries. The brachial plexus is a potential territory for absorption of local anesthetics. Analgesics prevent the generation and conduction of a nerve impulse, with a blockage between the origin of the impulse and the brain (Helbling and Guyer, 2016).

The visualization of structures by ultrasonography can be performed by means of longitudinal or transverse cuts, and it is preferable, for the blocking of peripheral nerves, the use of transversal cuts. In cross sections, it is possible to visualize the needle, positioning it parallel to the transducer, which allows to follow the progression of the tip and the body of the needle in the tissues, seen as a hyperechoic line. When the needle is positioned perpendicular to the transducer, it is possible to visualize only a cross-section of the needle, seen as a hyperechoic point (Helbling and Guyer, 2016).

The transducers used can be convex, have a larger field of view, or be linear, with better resolution of the image and more used in the visualization of surface structures. Low frequency ( 2 to $5 \mathrm{MHz}$.) transducers are ideal for viewing deep structures, while high frequency ( 6 to $15 \mathrm{MHz}$.) transducers display surface structures because they have limited penetration power in the tissues $(3$ to $4 \mathrm{~cm}$.). Thus, by decreasing the ultrasound frequency, the resolution of the images is also reduced. However, the power of penetration in the tissues increases, promoting the visualization of deep structures. As we increase the frequency of ultrasound, the sharpness of surface structures also increases, disfavours the deeper structures (Hemami et al., 2016).

Then, it becomes necessary to visualize the nerve and related anatomical structures. Hypoechoic structures, represented by dark areas (eg blood vessels, cysts, and water-rich tissues) reflect less the waves of the ultrasound beam. Hyperechoic structures, represented by white areas (eg bones, pleura, fat, tendons) reflect more the sound waves transmitted by the transducer than the adjacent structures.

The electrical signals, which are produced and processed by the transducer crystals, return as echoes to the transducer and then to the monitor where they are analyzed according to their strength and amplitude. The calculation of the distance traveled by the sound is made based on the time the wave takes to reach the object, to reflect and to return to the transducer. The longer the sound takes to reach the transducer, the further away the object is.

The returned echo is transformed into an electric impulse by the crystal, sent to an amplifier and shown on the monitor, with proportional intensities to its energy (Kim, 2017; Gonçalves et al., 2015).

\section{THE UNIVERSE OF MEDICAL SIMULATION}

The anesthesiological education through simulation has been proposed mainly for training, analysis and interpretation of phenomena, with the purpose of enabling the health professional to respond faster and eventual incidents.

Currently, the simulators are divided into two areas, but there are simulators that simultaneously incorporate the two:

- Software-based simulators, where the functions of the equipment and procedures are based on virtual reality, being simulated through images on a screen;

- Hardware-based simulators, where training is done on mannequins equipped with sensors where the goal is to simulate human patients in a simulated reality.

- Virtual reality in the area of medicine is divided into three groups:

- Planning: Allows a study based on a virtual replica of the actual situation, generated by images originating from magnetic resonance or computerized tomography of patients;

- Assistance: In this group, this technique is used as a resource to a real procedure, adding virtual elements to a real situation; 


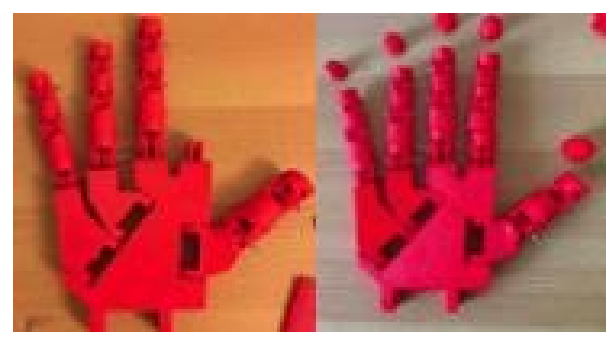

Figure 1. InMoov's hand printed for application in this project

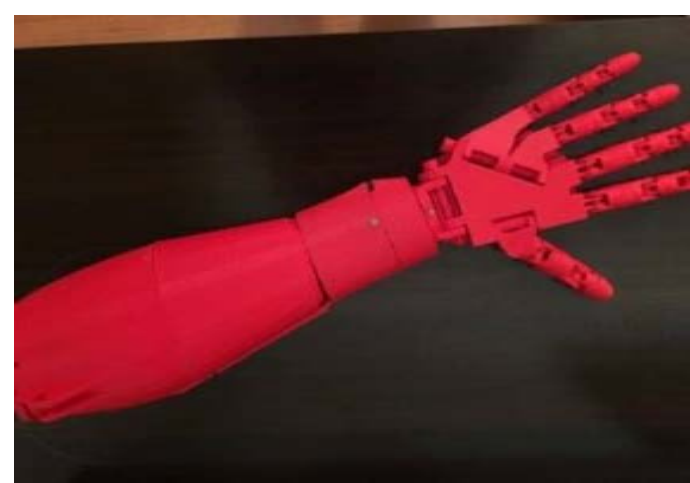

Figure 2. InMoov hand printed for application in this project

- Training: Uses the virtual environments to prepare the health professional to perform a certain procedure, and can simulate a situation with a high degree of realism (Niazi et al., 2012).

In simulated reality, the training is more directed to the human physiognomy. The development of these simulators is based on the anatomy of the body and its physiological reactions having a complete dummy with the structure of the human body or just the structures necessary for the training.

These types of simulators are the most common to find, with simulators with only a few anatomical structures to complete dummy simulators. These simulators are found in vendors such as SEEMSREAL (http://seemsreal.com/pt), Civiam (http://www.civiam.com.br/civiam) and 3BScientific (https://www.3bscientific.es).

The importance of simulation is the development of medical skills for situations where experience is needed, and the acquisition of this experience through training in patients is substituted.

In this simulator, the importance of the training consists in the differential movement of the two hands and in the procedure based on the images emitted in the monitor through the transducer.

\section{DEVELOPMENT OF THE PROTOTYPE}

In order to aid the training of health professionals, reducing possible risks in the surgical environment, an anesthetic simulator was developed, where it is possible to visualize the motor response, when the nerves of the brachial plexus are stimulated through its contact with the needle. Using action-research techniques, it is intended to validate whether the human nerve response can be effectively replicated.

In the context of the InMoov concept, the robot, a project open to the community that has better characteristics, and after downloading the STL files on its website, the members interested in the above project were printed: the hand and the forearm. It's important to note the fact that printed model already has all the holes necessary for the insertion of the nerves that will later be used in the different movements necessary for the simulation.

Figure 1 shows the pre- and post-construction of the hand.

After an impression of the hand, he proceeded to an impression of all the parts for a forearm assembly.

Figure 2 is illustrated a hand as well as the forearm, already with the joints made.

Since the anatomical structures were printed, it was necessary to use materials to simulate the human nerves. The material chosen was the fishing wire, due to its high strength and transparent color, it was also the material that the InMoov website referred to.

The fishing wire has been inserted into the nerve holes, from the fingertips to the forearm, where

Connected to the servomotors, as we can see in Figure 3. 


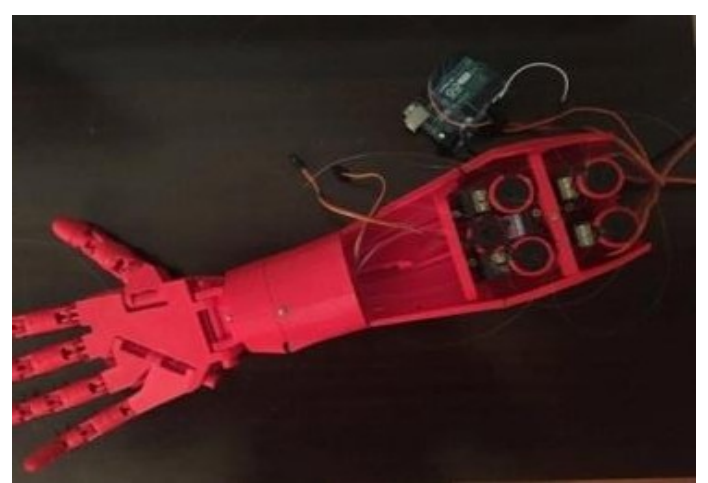

Figure 3. Adapter of the servomotors and connection to the Arduino board

For the programming of finger movements, the Arduino was chosen for its simplicity of technological implementation. With this environment, it was possible to recreate the nerve response, in the form of movement, that each nerve has when subjected to the action of the neurotransmitter needle, used in local anesthesia.

It is the Arduino board that controls the servomotors. These have gears and a shaft that can be controlled. Standard servomotors allow the axle to be positioned at various angles in the range of $0^{\circ}$ to $180^{\circ}$, while the continuously driven servomotors allow the shaft to rotate at various speeds. The servomotors used were SG5010, although they are not those that are referenced by InMoov. Since referrals were not available in Europe, they were chosen because they have the same binary and characteristics.

A servomotor is a small device whose shaft can be positioned in a given angular position according to a coded signal sent to it. As long as this code persists at the input, the servomotor will maintain its angular position. If the input code varies the angular position also varies (Paccola, 2014),

Servomotors have three wires: brown or black, red and yellow. The black or brown wire is the ground wire and is connected to a ground pin on the board (GND). The red wire is the power wire that must be connected to pin $5 \mathrm{~V}$ of the board. The yellow wire is the terminal of the signal that is connected to a digital pin of the board, this being the output pin of the servomotor (Ueshima et al., 2016).

The servomotors are connected to a door and have an associated input, as each movement recreated by the simulator involves more than one finger, where these are individually controlled by servomotors, it was necessary to group the servomotors by motion.

The simulator simulates two ideals motor responses, in one of them there is movement of the five fingers whereas in the other there is movement of three fingers. In this way, the servomotors were grouped into two groups:

- In the movement of only three fingers, myservo.attach (3), there is only one input but that originates in the movement of three fingers;

- In the movement of the five fingers, myservo1.attach (9), there is only one input but that originates in the movement of five fingers.

With this, whenever there is reception in the input associated with that servomotor, it starts the movement that is described in the code. These movements were against the human nervous response.

Each input is associated with a set of servomotors that perform their movement together. There are two inputs, in one of them there is movement of three servomotors that in turn lead to the movement of three fingers. In the other there is the movement of five servomotors and turn of five fingers.

When anesthesia is administered in the elbow area, the nerves under the action of the neurotransmitter needle are the median nerve, the ulnar nerve, and the radial nerve. Each nerve has a different response. The median nerve has an ideal motor response, flexing the fingers over the wrist and contracting four fingers (from the second to the fifth finger, beginning with the index finger). This movement is easy to reproduce as there is only direct action on the fingers.

The ulnar nerve has the ideal motor response, ulnar flexion of the wrist and contraction of the fourth and fifth fingers. In this movement, it was only possible to reproduce the contraction of the fingers, the printed model does not allow the ulnar flexion movement of the wrist.

The radial nerve has as ideal motor response the extension of the wrist and extension from the first to the fifth. This movement becomes less noticeable in the prototype.

\section{RESULTS AND CONCLUSION}

The importance of training in simulation has been considered over the years with the creation of simulators adapted to reality and meeting the needs of healthcare professionals. 


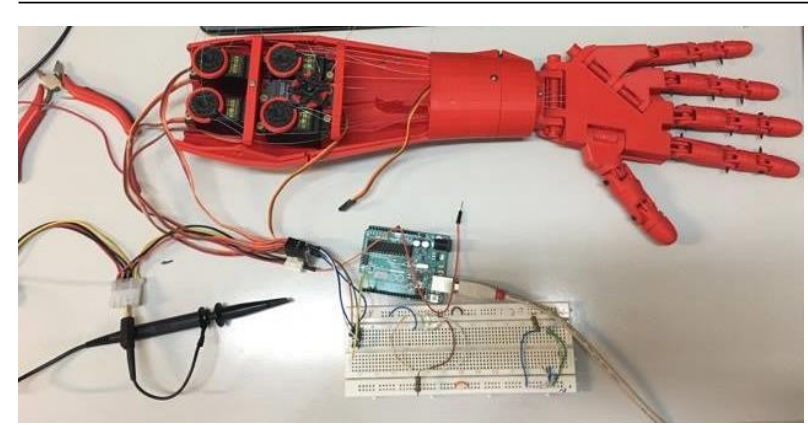

Figure 4. Simulator arm connected to the board

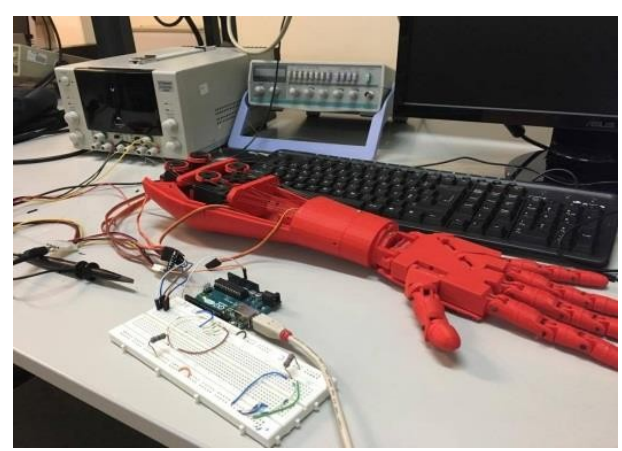

Figure 5. Power supply supplying power to the Arduino board

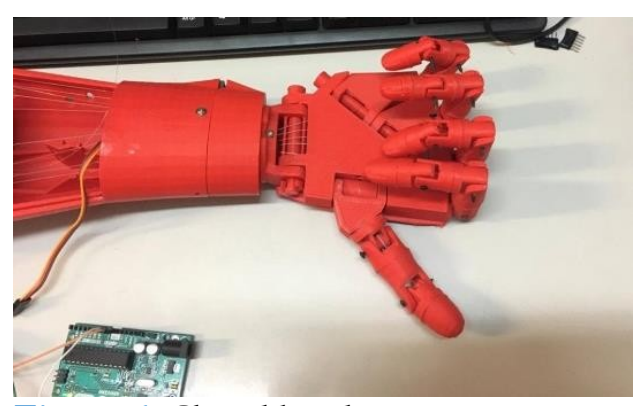

Figure 6. Closed hand

Proper training allows for quick responses, skill development, and improved technique and dexterity with the material. This reduces the risk to the patient.

With the development of the simulator, observed in Figure 4, it is possible to provide the health professional with a training tool that allows him to gain greater skill with the neurotransmitter needle while handling the transducer, guiding himself only in the monitor.

The ideal motor response when the neurotransmitter needle approaches the nerve is simulated by the movement of the fingers when there is the touch of the needle in the nerves in the anatomical region of the elbow. This movement is caused by the rotation of the servomotors at a certain angle and at a certain speed controlled by the Arduino, the board is powered by an external power source, as we saw in Figure 5.

The Arduino board was connected to a $5 \mathrm{~V}$ power supply. The power supply generates the power required to power the servomotors. These are fed with $5 \mathrm{~V}$ and receive the pulse width modulation (PWM) signal.

The servomotor control circuit is monitoring this signal at $20 \mathrm{~ms}$. intervals. If, in this interval of time the control circuit detects a change in the signal width, it changes the position of the axis so that its position coincides with the received signal, there being an impulse. This impulse is translated in the movement of the hand, as shown in Figure 6.

If the potentiometer is not in the position corresponding to the received signal, the control circuit drives the motor until the potentiometer is in the correct position. The motor will rotate in the direction that takes the potentiometer faster to the correct position. This rotating movement causes the move of the fingers. The entire joint system of the fingers is joined by the fishing wire, which in turn are connected to the servos, each finger individually. When the servo detects the change in signal width, it rotates, pulling the fishing wire that articulates the finger, when the servo returns to the initial position, another wire is pulled that performs the opposite movement at the finger joint. Performing the movements of flexion and extension of the fingers.

The flexion or extension movement of the fingers originates, when there is reception of the input that changes the width of the signal, the potentiometer moves to the corresponding position of the received signal. 


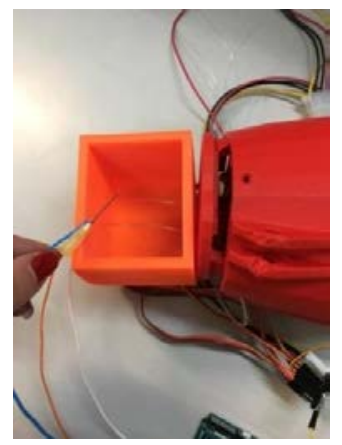

Figure 7. Simulator elbow

The simulator inputs are elbow region nerves that are simulated by two wires as seen in Figure 7, these wires are touched by the needle. Each copper wire corresponds to a different input, which leads to different movements in the fingers. The movement in the fingers is only carried out when the needle is in contact with one of the nerves, when there is no such contact the hand returns to its initial position.

The possibility of complications when performing anesthetic blocks justifies the use of ultrasonic assistance, which must be developed before blocking patients, so the importance of simulators, thus increasing the skill and confidence of health professionals.

The training is not only about the skill of handling the needle near the nerves, without any kind of contact. But also in the coordination between the two hands. Knowing that, they are concentrated in two different movements, while one hand gives us the anatomical images the other hand has to absorb this information and move to the desired places, the health professional needs a lot of training and precision in the hand.

The simulators come to help in this field, thus avoiding that the training is done in animals or materials adapted for the purpose that are only used once.

\section{REFERENCES}

Backus, T.C., Solounias, N. and Mihlbachler, M.C. (2016). The brachial plexus of the sumatran rhino (dicerorhinus sumatrensis) and application of brachial plexus anatomy toward mammal phylogeny. Journal of Mammalian Evolution, 23(1), pp. 71-79.

Carvalho, C.P. (2016). Project of robotic cell for handling. Revista de Gestão \& Tecnologia, 3(3).

Gonçalves, J., Faria, B.M., Reis, L.P., Carvalho, V. and Rocha, Á. (2015). Data mining and electronic devices applied to quality of life related to health data. In Information Systems and Technologies (CISTI), 2015 10th Iberian Conference on (pp. 1-4). IEEE.

Helbling, C. and Guyer, S.Z. (2016). Juniper: a functional reactive programming language for the arduino. In Proceedings of the 4th International Workshop on Functional Art, Music, Modelling, and Design, pages 8-16. ACM.

Hemami, H., Tarr, E., Li, B., Krishnamurthy, A., Clymer, B. and Dariush, B. (2016). Towards a cybernetic model of human movement. Mechanical Engineering Research, 6(1), 29.

Kim, L.T. (2017). Principles of ultrasound and applied ultrasound physics relevant for advanced sonographers. In Advanced Thyroid and Paratbyroid Ultrasound, pp. 37-47. Springer.

Niazi, A.U., Haldipur, N., Prasad, A.G. and Chan, V.W. (2012). Ultrasound- guided regional anesthesia performance in the early learning period: effect of simulation training. Regional anesthesia and pain medicine, 37(1), pp. 51-54.

Paccola, S.A.d.O. (2014). Design aplicado em simulação de anestesia infiltrativa bloqueio do nervo alveolar inferior. $\mathrm{PhD}$ thesis, Universidade de São Paulo.

Ueshima, H., Yoshiyama, S. and Otake, H. (2016). Probe's fixation during ultrasound-guided central venous puncture by using the echosupport. Journal of Clinical Anesthesia, 34, pp. 204-205. 